\title{
Resposta à aplicação e recuperação de enxofre em cultivos de casa de vegetação em solos com diferentes teores de argila e matéria orgânica
}

\author{
Responses to sulfur application and recuperation in greenhouse crops in soils with different clay and \\ organic matter content
}

\author{
Danilo dos Santos RheinheimerI Jimmy Walter Alvarez Rasche ${ }^{\text {II }}$ Benjamin Dias Osorio Filho ${ }^{\text {III }}$ \\ Leandro Souza da Silva
}

\section{RESUMO}

A dinâmica e a disponibilidade do enxofre têm sido pouco estudadas em relação a outros nutrientes, apesar da sua importância como nutriente à planta. Este estudo teve como objetivo avaliar a resposta de algumas culturas à aplicação de sulfato ( $\left.\mathrm{S}-\mathrm{SO}_{4}^{-2}\right)$ em solos com diferentes teores de argila e matéria orgânica, bem como avaliar a recuperação de enxofre, através de um balanço deste elemento no solo e na planta. O experimento consistiu de seis cultivos sucessivos (canola, soja, feijoeiro, gergelim, trevo vesiculoso e trigo), em vasos em casa de vegetação, utilizando-se quatro tipos de solos e quatro doses de $\mathrm{S}-\mathrm{SO}_{4}{ }^{-2}$, com quatro repetições distribuídas ao acaso. Avaliou-se a produção de matéria seca, o teor e o acúmulo de enxofre absorvido pelas culturas. Amostras de solo foram coletadas na camada de $0-10 \mathrm{~cm}$ após cada cultivo, para determinação do teor de $\mathrm{S}_{-} \mathrm{SO}_{4}^{-2}$ disponível. Dentre as culturas estudadas, somente a canola respondeu à aplicação de $\mathrm{S}_{-} \mathrm{SO}_{4}^{-2}$. Para as demais culturas, não houve relação entre os teores de $\mathrm{S}-\mathrm{SO}_{4}^{-2}$ da camada de $0-10 \mathrm{~cm}$ e a produção de matéria seca. Solos com diferentes teores de argila e matéria orgânica tiveram comportamento similar em relação à resposta das culturas à fertilização com enxofre; entretanto, a disponibilidade deste nutriente foi maior nos solos mais argilosos.

Palavras-chave: adubação sulfatada, disponibilidade de sulfato.

\section{ABSTRACT}

The sulfur (S) dynamic and availability is less studied than others nutrients, even though $S$ is an essential nutrient for crops production. This study was carried out to evaluate the crop responses to $\mathrm{S}_{-} \mathrm{SO}_{4}^{-2}$ application in different soils and to study $S$ recuperation by balancing it in soil and plants. The study was composed by a greenhouse experiment with six successive crops (canola, soybean, black bean, sesame, clover and wheat) using four soils and four $\mathrm{S}_{-} \mathrm{SO}_{4}^{-2}$ levels $(0,5$, 10 and $20 \mathrm{mg} \mathrm{kg}^{-1}$ ) to evaluate immediate and residual effect of $\mathrm{S}_{-\mathrm{SO}_{4}}^{-2}$ application. Soil samples were taken from $0-10 \mathrm{~cm}$ layer before and after each crop and analyzed for $\mathrm{S}_{-} \mathrm{SO}_{4}^{-2}$. It was evaluate the dry matter production and $\mathrm{S}_{-} \mathrm{SO}_{4}^{-2}$ absorbed by plants. Only canola responded to sulphur application. Soybean, black bean, sesame, clover and wheat did not response to sulfur application. There was not observed correlation between soil $\mathrm{S}_{-} \mathrm{SO}_{4}^{-2}$ available in $10 \mathrm{~cm}$ topsoil and crop yields. Soils with different clay and organic matter content showed similar behavior of crop response to sulfur application. The increase of $\mathrm{S}_{-} \mathrm{SO}_{4}^{-2}$ availability with fertilization was greater in soils with more clay content.

Key words: sulfur application, sulfate availability.

\section{INTRODUÇÃO}

O enxofre (S) é um nutriente essencial às plantas, pois participa na formação de diversas moléculas orgânicas, tais como os aminoácidos cisteína, cistina e metionina, que compõem a maioria das proteínas, as vitaminas biotina e tiamina, a coenzima A, entre outras (LARBIER \& LECLERCQ, 1992). Esse elemento encontra-se no solo na forma orgânica e inorgânica. Apesar de a primeira representar mais de $90 \%$ do S total na maioria dos solos (SOLOMON et al., 2005), é a forma inorgânica (ânion sulfato - $\mathrm{SO}_{4}^{-2}$ ) que é absorvida pelas plantas, a qual se encontra na solução do solo ou adsorvido na superfície dos colóides

\footnotetext{
IDepartamento de Solos, Universidade Federal de Santa Maria (UFSM), 97105-900, Santa Maria, RS, Brasil. E-mail: danilo@ccr.ufsm.br. Autor para correspondência.

"Universidade Nacional de Assunção. Assunção, Paraguai.

IIIPrograma de Pós-graduação em Ciência do Solo, UFSM, Santa Maria, RS, Brasil.
} 
inorgânicos por diferentes graus de energia, com ligação mono ou bi coordenada ao $\mathrm{Fe}$ ou Al (NEPTUNE et al., 1975; FRENEY et al., 1975, PARFITT \& SMART, 1978; BISSANI, 1985; CASAGRANDE et at., 2003).

A adsorção de $\mathrm{SO}_{4}^{-2}$ nos grupos funcionais dos colóides inorgânicos é dependente do $\mathrm{pH}$ do solo (CHAO et al., 1962; ELKINS \& ELMINGER, 1971; KORENTAJER et al., 1983), e em, solos com baixo conteúdo de colóides inorgânicos, a alta disponibilidade de $\mathrm{SO}_{4}^{-2}$ pode significar maiores perdas deste ânion por percolação (ERIKSEN \& ASKEGAARD, 2000). Dessa forma, os teores de $\mathrm{S}_{-} \mathrm{SO}_{4}^{-2}$ disponíveis estão relacionados com o $\mathrm{pH}$ do solo e os teores de matéria orgânica, argila e óxidos, e a maior probabilidade de respostas à adubação sulfatada ocorre em solos com pH elevado, baixos conteúdos de argila e matéria orgânica e que recebem cultivo intensivo associado ao uso de fertilizantes concentrados sem $\mathrm{S}$ e a uma contínua exportação deste elemento pelas colheitas.

Nas regiões produtoras de grãos do Estado do Rio Grande do Sul (Brasil), por muitos anos, foi utilizado o sistema convencional de preparo do solo, o qual provocou drástica redução nos teores de matéria orgânica e, conseqüentemente, na quantidade de $\mathrm{S}$ desses solos, provocada tanto pela mineralização quanto pela erosão. Atualmente, mesmo com a adoção do sistema de plantio direto, os incrementos nos teores de matéria orgânica são lentos em decorrência da baixa quantidade de resíduos produzidos anualmente, quer por causa da manutenção de pousio invernal, quer pelo monocultivo de soja no verão, ou até pela combinação das duas situações (RHEINHEIMER et al., 2001). Isso significa que está ocorrendo imobilização de enxofre na matéria orgânica. Como a produtividade média das culturas, especialmente da soja, também tem aumentado lentamente (MIELNICZUK et al., 2000) e a reposição de enxofre não deve estar ocorrendo com a adição de fertilizantes concentrados em NPK ERIKSEN \& ASKEGAARD (2000) alertam para o fato de que a as perdas de sulfato por percolação podem tornar negativo o balanço entre entradas e saídas de enxofre no solo, independentemente do tipo de rotação de culturas adotado. Se for considerado que as deposições atmosféricas desse nutriente tendem a diminuir no futuro, haverá necessidade de reposição de fertilizantes sulfatados, para, no mínimo, tornar o balanço nulo. Desse modo, acredita-se que possam haver respostas à fertilização com $\mathrm{S}$, pelo menos para aquelas culturas mais exigentes.

$\mathrm{O}$ diagnóstico da disponibilidade de $\mathrm{S}_{-} \mathrm{SO}_{4}^{-2}$ nos Estados do Rio Grande do Sul e de Santa Catarina é feito pela quantificação de $\mathrm{S}_{-} \mathrm{SO}_{4}^{-2}$ extraído por $\mathrm{Ca}\left(\mathrm{H}_{2} \mathrm{PO}_{4}\right)_{2}$. O nível de suficiência considerado atualmente é de $10 \mathrm{mg} \mathrm{dm}^{-3}$ de $\mathrm{S}_{-} \mathrm{SO}_{4}^{-2}$ para culturas exigentes como brássicas, liliáceas e leguminosas, e de $5 \mathrm{mg} \mathrm{dm}^{-3} \mathrm{de} \mathrm{S} \mathrm{SO}_{4}^{-2}$ para os demais grupos de culturas (CQFS-RS/SC, 2004). Entretanto, os experimentos com fertilização sulfatada são relativamente escassos nesses Estados (GOEPFERT \& KUSSOW, 1971; ANGHINONI, 1975, ANGHINONI et al., 1976; COSTA, 1980; NASCIMENTO \& MORELLI, 1980; WOLFFENBÜTTEL \& TEDESCO, 1981; BISSANI, 1985). Além disso, os resultados obtidos em outros locais são contraditórios, dificultando o estabelecimento do nível crítico, das classes de disponibilidade, e, conseqüentemente, das recomendações adequadas de fertilizantes sulfatados. Assim, há necessidade de se avaliar a disponibilidade de $\mathrm{S}$ em solos com diferentes teores de argila e matéria orgânica e realizar ensaios na tentativa de melhorar a definição do nível crítico e da resposta das plantas.

O objetivo deste trabalho foi avaliar (a) a produção de matéria seca da parte aérea e a quantidade de enxofre absorvido por algumas culturas em decorrência da aplicação de sulfato em quatro solos do Rio Grande do Sul com diferentes teores de argila e matéria orgânica e (b) os teores de sulfato do solo após cultivos sucessivos.

\section{MATERIAL E MÉTODOS}

O experimento foi conduzido em casa de vegetação, em vasos, no Departamento de Solos da Universidade Federal de Santa Maria (UFSM), consistindo de uma sucessão das culturas canola (Brassica napus L. var. napus), soja (Glycine max L.), feijoeiro (Phaseolus vulgaris L.), gergelim (Sesamum indicum L.), trevo vesiculoso (Trifolium vesiculosum cv. Yuchi) e trigo (Triticum aestivum L.). Utilizou-se o delineamento inteiramente casualizado, em esquema bifatorial, com quatro repetições. $\mathrm{O}$ primeiro fator consistiu de quatro tipos de solos com diferentes teores de argila e matéria orgânica: Neossolo Quatzarênico Órtico típico (RQo), sob campo nativo e coletado em Manuel Viana-RS; Argissolo Vermelho Distrófico arênico (PVd), sob sistema de plantio direto há 14 anos e coletado em Santa Maria-RS; Latossolo Vermelho-Amarelo Distrófico típico (LVAd) sob sistema de plantio direto há 8 anos e coletado em Júlio de Castilhos-RS; e um Latossolo Vermelho Distroférrico típico (LVdf) sob sistema de plantio direto há 8 anos e coletado em Ibirubá-RS (Tabela 1). As amostras de solo foram coletadas indeformadas com tubos de PVC de $200 \mathrm{~mm}$ de diâmetro e $220 \mathrm{~mm}$ de profundidade e acondicionadas em vaso com, aproximadamente, $11 \mathrm{~kg}$ de solo. Para caracterizar quimicamente os quatro solos, determinou-se o $\mathrm{pH}$ em água, índice SMP e teores 
Tabela 1 - Caracterização química dos solos utilizados.

\begin{tabular}{|c|c|c|c|c|c|c|c|c|c|c|}
\hline Solos $^{1}$ & argila & m.o. & $\mathrm{pH}_{\mathrm{H} 2 \mathrm{O}}$ & SMP & $\mathrm{S}^{2}$ & $\mathrm{P}^{3}$ & $\mathrm{~K}^{3}$ & $\mathrm{Ca}^{4}$ & $\mathrm{Mg}^{4}$ & $\mathrm{Al}^{4}$ \\
\hline & \multicolumn{2}{|c|}{$\ldots \ldots \mathrm{g} \mathrm{dm}^{-3} \ldots .}$. & $1: 1$ & & \multicolumn{3}{|c|}{$\ldots \ldots \ldots \ldots \mathrm{mg} \mathrm{dm}^{-3} \ldots \ldots \ldots \ldots \ldots$} & \multicolumn{3}{|c|}{.................. $\mathrm{cmol}_{\mathrm{c}} \mathrm{dm}^{-3} .}$. \\
\hline LVdf & 640 & 32,0 & 6,6 & 6,8 & 11,4 & 4,1 & 96 & 7,53 & 2,00 & 0 \\
\hline LVAd & 320 & 27,2 & 5,1 & 5,6 & 13,6 & 9,8 & 102 & 2,38 & 0,73 & 0,65 \\
\hline PVd & 140 & 29,3 & 5,3 & 5,8 & 9,3 & 18,8 & 93 & 2,71 & 0,62 & 0,15 \\
\hline RQo & 80 & 6,7 & 4,7 & 6,1 & 4,8 & 8,0 & 30 & 0,52 & 0,15 & 0,20 \\
\hline
\end{tabular}

${ }^{1}$ Latossolo Vermelho-Amarelo Distrófico típico (LVAd); e Latossolo Vermelho Distroférrico típico (LVdf); Argissolo Vermelho Distrófico arênico (PVd) e Neossolo Quatzarênico Órtico típico (RQo).

${ }^{2}$ extraído por $\mathrm{Ca}\left(\mathrm{H}_{2} \mathrm{PO}_{4}\right)_{2} ;{ }^{3}$ extraído por Mehlich $1 ;{ }^{4}$ extraído por $\mathrm{KCl} 1 \mathrm{~mol} \mathrm{~L}^{-1}$

disponíveis de $\mathrm{S}, \mathrm{P}, \mathrm{K}, \mathrm{Ca}, \mathrm{Mg}$ e $\mathrm{Al}$ por extratores específicos (Tabela 1), de acordo com TEDESCO et al. (1995). Antes do primeiro cultivo, adicionou-se calcário na metade da dose equivalente para elevar o $\mathrm{pH}$ a 6,0 no solo RQo e uma dose inteira para elevar o $\mathrm{pH}$ a 6,0 no solo LVAd.

Para o outro fator, usaram-se quatro tratamentos com aplicação de sulfato da seguinte maneira: aplicação de sulfato, no primeiro cultivo, em doses equivalentes a $0,55,110$ e $220 \mathrm{mg}$ de $\mathrm{S}_{-} \mathrm{SO}_{4}$ vaso $^{-1}$; reaplicação de um terço dessas doses no segundo cultivo e sem reaplicação de sulfato no terceiro cultivo. Repetiu-se esse esquema nos três cultivos seguintes (aplicação das doses completas no quarto cultivo, um terço das doses no quinto cultivo e sem reaplicação de sulfato no sexto cultivo). Após os seis cultivos sucessivos, os quatro tratamentos com sulfato receberam o equivalente a $0 ; 146,6 ; 293,4$ e 586,6mg de $\mathrm{S}_{-} \mathrm{SO}_{4}$ vaso $^{-1}$ (Tabela 2). Na implantação dos cultivos, adicionou-se solução nutritiva contendo $30 \mathrm{mg}$ de $\mathrm{N}$ $\mathrm{kg}^{-1}$ de solo (nas culturas não-leguminosas) na forma de $\left(\mathrm{NH}_{4}\right)_{2} \mathrm{SO}_{4}$ ou $\mathrm{NH}_{4} \mathrm{NO}_{3} ; 100 \mathrm{mg} \mathrm{de}^{\mathrm{P} \mathrm{kg}}{ }^{-1}$ de solo na

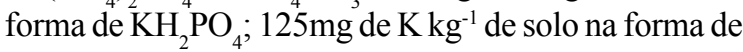
$\mathrm{KH}_{2} \mathrm{PO}_{4}$ ou K $2 \mathrm{SO}_{4} ; 2 \mathrm{mg}$ de $\mathrm{Zn} \mathrm{kg}^{-1}$ de solo na forma de $\mathrm{ZnCl}_{2} ; 1 \mathrm{mg}$ de $\mathrm{Cu} \mathrm{kg}^{-1}$ de solo na forma de $\mathrm{CuCl}_{2} \mathrm{e}$ $0,5 \mathrm{mg}$ de $\mathrm{B} \mathrm{kg} \mathrm{kg}^{-1}$ de solo na forma de $\mathrm{H}_{3} \mathrm{BO}_{3}$. Para adubação nitrogenada de cobertura, foi utilizado $\mathrm{NH}_{4} \mathrm{NO}_{3}$. Antes do cultivo do feijoeiro e do trevo vesiculoso, foram adicionados $0,2 \mathrm{mg}$ de $\mathrm{Mn} \mathrm{kg}^{-1} \mathrm{de}$ solo na forma de $\mathrm{MnCl}_{2} \cdot 4 \mathrm{H}_{2} \mathrm{O}$ e $0,01 \mathrm{mg}$ de $\mathrm{Mo} \mathrm{kg}^{-1} \mathrm{de}$ solo na forma de $\left(\mathrm{NH}_{4}\right)_{6} \mathrm{Mo}_{7} \mathrm{O}_{24} \cdot 4 \mathrm{H}_{2} \mathrm{O}$. As doses de S$\mathrm{SO}_{4}^{-2}$ foram aplicadas na superfície do solo e a fonte de $\mathrm{S}$ foi o $\left(\mathrm{NH}_{4}\right)_{2} \mathrm{SO}_{4}$ nas culturas que receberam nitrogênio na semeadura e $\mathrm{K}_{2} \mathrm{SO}_{4}$ nas leguminosas, que, por terem sido inoculadas com bactérias fixadoras de nitrogênio, não receberam adubação nitrogenada.

A primeira cultura foi a canola, utilizando-se sementes pré-germinadas. Logo após a emergência, realizou-se desbaste, deixando-se 10 plantas por vaso e, 18 dias após a emergência, aplicaram-se $20 \mathrm{mg}$ de N $\mathrm{kg}^{-1}$ de solo. A segunda cultura semeada foi a soja (variedade RS-10), deixando-se nove plantas por vaso. O terceiro cultivo foi o feijoeiro, deixando-se oito plantas por vaso, sendo que, 18 dias após a emergência, aplicaram-se $30 \mathrm{mg}$ de $\mathrm{N} \mathrm{kg}^{-1}$ de solo. Como quarto cultivo, utilizou-se o gergelim, deixando-se quatro

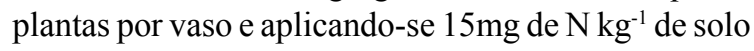
aos 15 dias após a emergência. $\mathrm{O}$ quinto cultivo semeado foi o trevo vesiculoso, deixando-se 10 plantas por vaso. Por fim, o sexto cultivo foi o trigo, deixandose 11 plantas por vaso e aplicando-se 10 e $20 \mathrm{mg}$ de $\mathrm{N}$ $\mathrm{kg}^{-1}$ de solo aos 10 e 20 dias após a emergência, respectivamente.

Em todos os cultivos, quando as plantas atingiram o pleno florescimento, a parte aérea foi cortada rente ao solo. A matéria seca foi determinada após a secagem em estufa a $60^{\circ} \mathrm{C}$ até peso constante, a qual foi moída e determinado o teor de $\mathrm{S}$ total, após a

Tabela 2 - Doses de S-SO ${ }_{4}^{-2}$ utilizadas em cada uma das seis culturas.

\begin{tabular}{|c|c|c|c|c|c|c|c|}
\hline \multirow{2}{*}{ Tratamento } & \multicolumn{7}{|c|}{ Culturas } \\
\hline & canola & soja & feijoeiro & gergelim & trevo vesicul. & trigo & total \\
\hline & \multicolumn{7}{|c|}{ 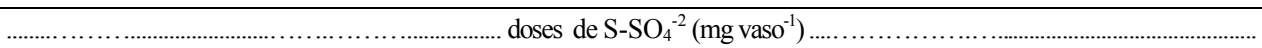 } \\
\hline $\mathrm{T} 1$ & 0 & 0 & 0 & 0 & 0 & 0 & 0 \\
\hline $\mathrm{T} 2$ & 55 & $+18,3$ & 0 & +55 & $+18,3$ & 0 & $=146,6$ \\
\hline $\mathrm{T} 3$ & 110 & $+36,7$ & 0 & +110 & $+36,7$ & 0 & $=293,4$ \\
\hline $\mathrm{T} 4$ & 220 & $+73,3$ & 0 & +220 & $+73,3$ & 0 & $=586,6$ \\
\hline
\end{tabular}

Ciência Rural, v.37, n.2, mar-abr, 2007. 
digestão nitro-perclórica (TEDESCO et al., 1995). A quantidade de $\mathrm{S}$ exportada pelas culturas foi estimada pelo teor de $\mathrm{S}$ no tecido da parte área e pela produção de matéria seca. Após todos os cultivos, foi coletada uma amostra de solo na camada de $0-10 \mathrm{~cm}$, a qual foi secada em estufa, moída e peneirada para determinação da disponibilidade de $\mathrm{S}-\mathrm{SO}_{4}^{-2}$ pelo extrator $\mathrm{Ca}\left(\mathrm{H}_{2} \mathrm{PO}_{4}\right)_{2}$ (TEDESCO et al., 1995).

Os resultados do rendimento de matéria seca, $\mathrm{S}_{-} \mathrm{SO}_{4}^{-2}$ disponível no solo, teor de $\mathrm{S}$ no tecido e quantidade de $\mathrm{S}$ exportada, para cada cultura separadamente, foram submetidos à análise de variância, num esquema bifatorial (quatro tipos de solos e quatro doses de sulfato). Quando houve significância estatística, as médias dos tratamentos qualitativos (tipo de solos) foram comparadas pelo teste de Tukey ao nível de 0,05 de probabilidade de erro e por ajustes de equações polinomiais para os tratamentos quantitativos (doses de sulfato).

\section{RESULTADOS E DISCUSSÃO}

A aplicação de $\mathrm{S}^{-\mathrm{SO}_{4}{ }^{-2}}$ no solo proporcionou incremento de matéria seca na cultura da canola, apesar de os teores de sulfato disponível estarem acima do nível de suficiência proposto pela CQFS-RS/SC (2004) (Tabela 3). Considerando o rendimento relativo (RR), média dos quatro solos, em relação ao teor de $\mathrm{S}_{-} \mathrm{SO}_{4}^{-2}$ disponível no solo, obtevese a equação:

$R R=100,71\left(1-e^{0,0901 \text { teor sulfato }}\right)$, resultando num

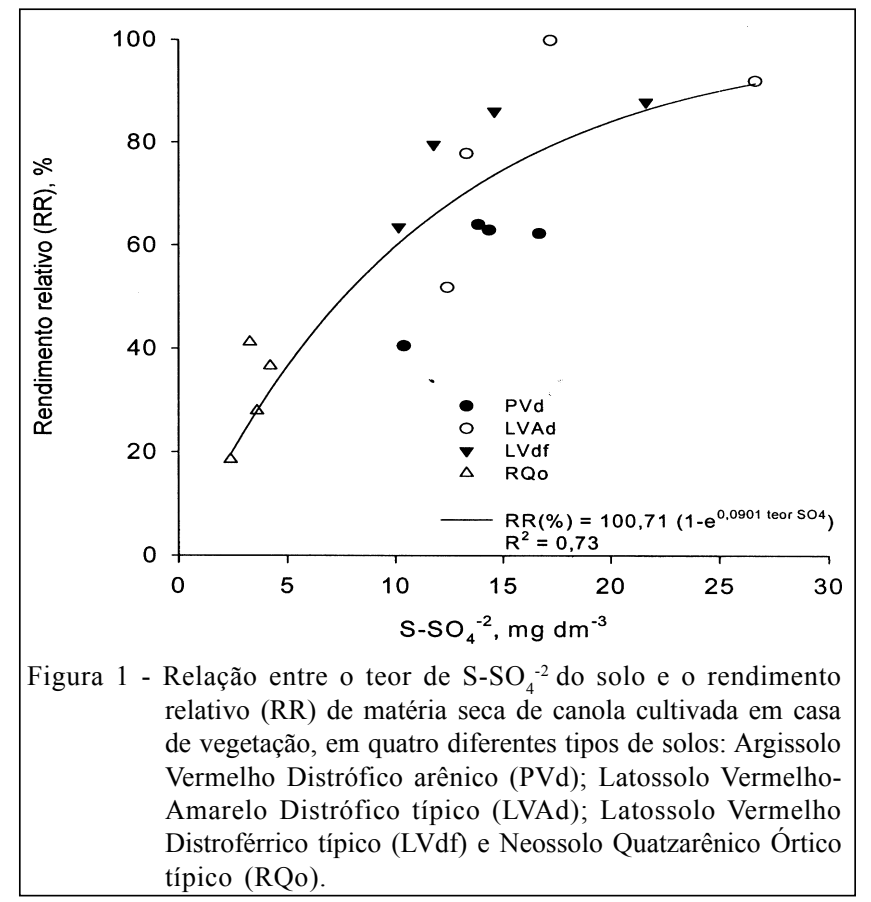

nível de suficiência de $24,8 \mathrm{mg}$ de $\mathrm{S}_{-} \mathrm{SO}_{4}^{-2} \mathrm{dm}^{-3}$ de solo (Figura 1). No entanto, deve-se enfatizar que resultados obtidos em casa de vegetação não são diretamente aplicáveis para recomendação de fertilização sulfatada em lavouras. Além de diferenças de produção de matéria seca em função das doses aplicadas, houve diferenças em função dos tipos de solos, sendo que o LVAd proporcionou maior produção de matéria seca de canola em relação aos solos PVd e RQo, não diferindo, porém, do outro latossolo (LVdf) (Tabela 3).

As culturas da soja, do feijoeiro, do gergelim, do trevo vesiculoso e do trigo não responderam à aplicação de $\mathrm{S}^{-\mathrm{SO}_{4}^{-2}}$ (Tabela 3). Entretanto, houve diferenças na produção de matéria seca destas culturas entre os solos. As diferenças de produção observadas entre as classes de solos não estão relacionadas à disponibilidade de $\mathrm{S}_{-} \mathrm{SO}_{4}^{-2}$, pois solos com semelhantes teores de $\mathrm{S}_{-} \mathrm{SO}_{4}^{-2}$ ( $\mathrm{LVdf}$ e $\mathrm{PVd}$ ) tiveram diferentes produções de matéria seca e solos com teores bastante distintos de $\mathrm{S}_{-} \mathrm{SO}_{4}^{-2}$ (PVd e RQo) tiveram produções semelhantes. Desse modo, a disponibilidade inicial de $\mathrm{S}^{-\mathrm{SO}_{4}}{ }^{-2}$ no solo foi suficiente para suprir adequadamente a produção de matéria seca da soja e do feijoeiro, mesmo quando o teor era menor do que o teor crítico definido pela CQFS-RS/SC (2004), como no caso do solo RQo.

A análise estatística dos teores de $\mathrm{S}^{-\mathrm{SO}_{4}^{-2}}$ extraídos pelo $\mathrm{Ca}\left(\mathrm{H}_{2} \mathrm{PO}_{4}\right)_{2}$ das amostras de solos coletados após o cultivo da canola mostrou interação significativa entre solos e doses de $\mathrm{S}$. Os teores de $\mathrm{S}$ no solo aumentaram com as doses de $\mathrm{S}_{-} \mathrm{SO}_{4}^{-2}$ (Tabela 3). A aplicação de enxofre nos três primeiros solos resultou em maior recuperação de $\mathrm{S}^{-\mathrm{SO}_{4}}{ }_{4}^{-2}$. No solo LVdf, os teores de S-SO ${ }_{4}^{-2}$ aumentaram linearmente com a aplicação de $\mathrm{S}$ $\mathrm{SO}_{4}^{-2}\left(\mathrm{~S}_{-} \mathrm{SO}_{4}^{-2}=9,44+0,584\right.$ dose; $\left.\mathrm{R}^{2}=0,98\right)$, sendo que, por cada $\mathrm{kg}$ de $\mathrm{S}_{-} \mathrm{SO}_{4}^{-2}$ aplicado no solo, $0,58 \mathrm{~kg}$ dele foi recuperado pelo $\mathrm{Ca}\left(\mathrm{H}_{2} \mathrm{PO}_{4}\right)_{2}$. A capacidade de acúmulo de sulfato no LVAd foi superior a do solo $\mathrm{LVdf}\left(\mathrm{S}_{-} \mathrm{SO}_{4}^{-2}=10,86+0,747\right.$ dose; $\mathrm{R}^{2}=0,95$ ), possivelmente pelo menor valor de $\mathrm{pH}$, sendo que, cada $\mathrm{kg}$ de $\mathrm{S}_{-} \mathrm{SO}_{4}^{-2}$ adicionado ao solo resultou em $0,74 \mathrm{~kg}$ recuperado na camada de $0-10 \mathrm{~cm}$ do solo. O acúmulo de sulfato no PVd $\left(\mathrm{S}_{-} \mathrm{SO}_{4}^{-2}=11,34+0,287\right.$ dose; $\left.\mathrm{R}^{2}=0,88\right)$ ocorreu de forma mais lenta que nos Latossolos, pois, de cada $\mathrm{kg}$ de $\mathrm{S}_{-} \mathrm{SO}_{4}^{-2}$ adicionado ao solo, somente $0,29 \mathrm{~kg}$ foi recuperado na camada de $0-10 \mathrm{~cm}$ do solo. Os dados de $\mathrm{S}^{-\mathrm{SO}_{4}{ }^{-2}}$ disponível no RQo não se ajustaram a nenhuma equação matemática. Este solo apresentou baixa capacidade de retenção de sulfato por conter baixos teores de argila, sendo, 
Tabela 3 - Disponibilidade de $\mathrm{S}^{-\mathrm{SO}_{4}}{ }^{-2}$ no solo e produção de matéria seca de seis cultivos em sucessão decorrente da aplicação das doses de enxofre em quatro diferentes tipos de solo.

\begin{tabular}{|c|c|c|c|c|c|c|c|c|}
\hline \multirow{3}{*}{$\begin{array}{l}\text { Doses } \\
\text { acumuladas de } \\
\mathrm{S}_{-} \mathrm{SO}_{4}^{-2} \\
\mathrm{mg} \mathrm{vaso}^{-1} \\
\end{array}$} & \multicolumn{2}{|c|}{$\mathrm{LVdf}^{1}$} & \multicolumn{2}{|c|}{ LVAd } & \multicolumn{2}{|c|}{$\mathrm{PVd}$} & \multicolumn{2}{|c|}{ RQo } \\
\hline & S-SO ${ }_{4}^{-2}$ solo & Matéria seca & $\mathrm{S} \mathrm{SO}_{4}^{-2}$ solo & Matéria seca & $\mathrm{S}-\mathrm{SO}_{4}^{-2}$ solo & Matéria seca & $\mathrm{S} \mathrm{SO}_{4}^{-2}$ solo & Matéria seca \\
\hline & $\mathrm{mg} \mathrm{dm}^{-3}$ & $\mathrm{~g} \mathrm{vaso}^{-1}$ & $\mathrm{mg} \mathrm{dm}^{-3}$ & g vaso $^{-1}$ & $\mathrm{mg} \mathrm{dm}^{-3}$ & $\mathrm{~g} \mathrm{vaso}^{-1}$ & $\mathrm{mg} \mathrm{dm}^{-3}$ & g vaso $^{-1}$ \\
\hline & \multicolumn{8}{|c|}{ } \\
\hline 0 & $10,2 \mathrm{~A}^{2}$ & 10,7 & $12,4 \mathrm{~A}$ & 8,7 & $10,4 \mathrm{~A}$ & 6,8 & $1,9 \mathrm{~B}$ & 3,1 \\
\hline 55 & $11,8 \mathrm{~A}$ & 13,4 & $13,3 \mathrm{~A}$ & 13,1 & $13,9 \mathrm{~A}$ & 10,8 & $3,6 \mathrm{~B}$ & 4,7 \\
\hline 110 & $14,6 \mathrm{~A}$ & 14,5 & $17,2 \mathrm{~A}$ & 16,8 & $14,4 \mathrm{~A}$ & 10,6 & $3,3 \mathrm{~B}$ & 6,9 \\
\hline 220 & $21,6 \mathrm{~B}$ & 14,8 & $26,7 \mathrm{~A}$ & 15,5 & $16,7 \mathrm{C}$ & 10,5 & $4,2 \mathrm{D}$ & 6,2 \\
\hline Média & & $13,3 a b^{3}$ & & $13,5 \mathrm{a}$ & & $9,7 \mathrm{~b}$ & & $5,2 \mathrm{c}$ \\
\hline $\mathrm{CV} \%$ & \multicolumn{8}{|c|}{ Matéria seca $=18,0$ e teor de $\mathrm{S}_{-} \mathrm{SO}_{4}{ }^{-2}=17,4$} \\
\hline 0 & $11,0 \mathrm{~A}$ & 9,6 & $9,1 \mathrm{~A}$ & 10,7 & $12,0 \mathrm{~A}$ & 8,3 & $1,8 \mathrm{~B}$ & 9,6 \\
\hline 73,3 & $12,4 \mathrm{~A}$ & 9,9 & $13,6 \mathrm{~A}$ & 9,3 & $13,7 \mathrm{~A}$ & 8,6 & $2,0 \mathrm{~B}$ & 9,0 \\
\hline 146,6 & $16,3 \mathrm{~B}$ & 8,9 & $21,8 \mathrm{~A}$ & 10,5 & $16,7 \mathrm{AB}$ & 9,1 & $2,4 \mathrm{C}$ & 8,4 \\
\hline 293,3 & $28,8 \mathrm{~A}$ & 9,4 & $26,5 \mathrm{AB}$ & 9,8 & $22,7 \mathrm{~B}$ & 9,4 & $3,9 \mathrm{C}$ & 8,6 \\
\hline Média & & $9,5 \mathrm{ab}$ & & $10,0 \mathrm{a}$ & & $8,9 \mathrm{~b}$ & & $8,9 \mathrm{~b}$ \\
\hline $\mathrm{CV} \%$ & \multicolumn{8}{|c|}{ Matéria seca $=11,6$ e teor de $\mathrm{S}_{-} \mathrm{SO}_{4}{ }^{-2}=27,9$} \\
\hline & & & & Feij & eiro.. & & & \\
\hline 0 & $9,4 \mathrm{~B}$ & 11,2 & $14,6 \mathrm{~A}$ & 11,9 & $10,6 \mathrm{AB}$ & 9,6 & $3,4 \mathrm{C}$ & 7,8 \\
\hline 73,3 & $10,9 \mathrm{~B}$ & 12,0 & $17,6 \mathrm{~A}$ & 11,6 & $11,0 \mathrm{~B}$ & 9,5 & $3,1 \mathrm{C}$ & 7,6 \\
\hline 146,6 & $16,5 \mathrm{~A}$ & 11,5 & $18,0 \mathrm{~A}$ & 11,5 & $9,4 \mathrm{~B}$ & 9,9 & $3,8 \mathrm{C}$ & 8,5 \\
\hline 293,3 & $28,8 \mathrm{~A}$ & 11,1 & $29,4 \mathrm{~A}$ & 11,5 & $12,0 \mathrm{~B}$ & 10,6 & $3,1 \mathrm{C}$ & 8,9 \\
\hline Média & & $11,5 \mathrm{a}$ & & $11,6 \mathrm{a}$ & & $9,9 \mathrm{~b}$ & & $8,2 \mathrm{c}$ \\
\hline $\mathrm{CV} \%$ & \multicolumn{8}{|c|}{ Matéria seca $=7,6$ e teor de $\mathrm{S}_{-} \mathrm{SO}_{4}{ }^{-2}=22,0$} \\
\hline 0 & 12,4 & 7,9 & 14,9 & $\begin{array}{l}\ldots \ldots \ldots \text { Ger } \\
6,9\end{array}$ & $\lim _{8,4} \ldots \ldots$ & 6,9 & 6,8 & 6,0 \\
\hline 128,3 & 14,8 & 8,3 & 17,7 & 7,0 & 14,6 & 6,0 & 10,1 & 5,6 \\
\hline 256,7 & 21,5 & 8,6 & 24,9 & 5,9 & 17,3 & 6,7 & 15,2 & 4,8 \\
\hline 513,3 & 34,8 & 8,3 & 35,1 & 7,7 & 27,0 & 6,0 & 19,5 & 5,6 \\
\hline Média & $20,6 \mathrm{~A}$ & $8,3 \mathrm{a}$ & $23,1 \mathrm{~A}$ & $6,7 \mathrm{~b}$ & $16,8 \mathrm{~B}$ & $6,4 \mathrm{~b}$ & $12,9 \mathrm{C}$ & $5,5 \mathrm{c}$ \\
\hline $\mathrm{CV} \%$ & \multicolumn{8}{|c|}{ Matéria seca $=16,7$ e teor de $\mathrm{S}_{-} \mathrm{SO}_{4}{ }^{-2}=24,4$} \\
\hline 0 & 20,7 & 9,6 & 14,4 & 10,7 & $\begin{array}{c}\text { vo......... } \\
11,1\end{array}$ & 8,3 & 9,5 & 9,6 \\
\hline 146,6 & 21,8 & 9,9 & 17,8 & 9,3 & 17,9 & 8,6 & 10,1 & 9,0 \\
\hline 293,4 & 26,4 & 8,9 & 19,2 & 10,5 & 12,6 & 9,1 & 15,2 & 8,4 \\
\hline 586,6 & 29,9 & 9,4 & 18,5 & 9,8 & 15,4 & 9,4 & 19,5 & 8,6 \\
\hline Média & $24,7 \mathrm{~A}$ & $9,5 \mathrm{ab}$ & $17,5 \mathrm{~B}$ & $10,0 \mathrm{a}$ & $14,2 \mathrm{C}$ & $8,9 \mathrm{~b}$ & $10,9 \mathrm{D}$ & $8,9 \mathrm{~b}$ \\
\hline $\mathrm{CV} \%$ & \multicolumn{8}{|c|}{ Matéria seca $=14,3$ e teor de $\mathrm{S}_{-} \mathrm{SO}_{4}{ }^{-2}=21,3$} \\
\hline 0 & $12,9 \mathrm{~A}$ & 10,1 & $10,0 \mathrm{AB}$ & 10,5 & 7,9 B & 10,0 & $4,0 \mathrm{C}$ & 9,8 \\
\hline 146,6 & $15,7 \mathrm{~A}$ & 10,7 & $10,8 \mathrm{~B}$ & 11,0 & $8,4 \mathrm{~B}$ & 12,0 & $4,2 \mathrm{C}$ & 11,0 \\
\hline 293,4 & $19,0 \mathrm{~A}$ & 10,6 & $13,5 \mathrm{~B}$ & 11,6 & $8,6 \mathrm{~B}$ & 10,3 & $5,0 \mathrm{C}$ & 10,9 \\
\hline 586,6 & $22,9 \mathrm{~A}$ & 11,4 & $17,9 \mathrm{~B}$ & 11,0 & $7,5 \mathrm{C}$ & 11,0 & $4,1 \mathrm{D}$ & 10,6 \\
\hline Média & & $10,7^{\mathrm{ns}}$ & & 11,0 & & 10,8 & & 10,6 \\
\hline CV\% & \multicolumn{8}{|c|}{ Matéria seca $=11,6$ e teor de $\mathrm{S}^{-\mathrm{SO}_{4}}{ }^{-2}=21,8$} \\
\hline
\end{tabular}

${ }^{1}$ Latossolo Vermelho-Amarelo Distrófico típico (LVAd); e Latossolo Vermelho Distroférrico típico (LVdf); Argissolo Vermelho Distrófico arênico (PVd) e Neossolo Quatzarênico Órtico típico (RQo).

${ }^{2}$ Médias dos teores de $\mathrm{S}_{-} \mathrm{SO}_{4}^{-2}$ seguidas pela mesma letra maiúscula na linha, comparando as classes de solo dentro de cada dose de $\mathrm{S}_{-} \mathrm{SO}_{4}{ }^{-2}$, não diferiram estatisticamente pelo teste de Tukey $(\alpha 5 \%)$.

${ }^{3}$ Médias da produção de matéria seca seguidas pela mesma letra minúscula na linha, comparando as classes de solo na média das doses, não diferiram estatisticamente pelo teste de Tukey ( $\alpha$ 5\%).

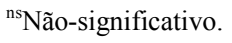


conseqüentemente, pobre em óxidos de ferro e de alumínio e argilominerais $1: 1$, além de o $\mathrm{pH}$ ser elevado $(6,6)$ e ter recebido altas doses de fósforo no momento de implantar a cultura. A reaplicação de $1 / 3$ da dose inicial no cultivo da soja foi suficiente para manter os níveis de $\mathrm{S}_{-} \mathrm{SO}_{4}^{-2}$ no solo, mesmo após o cultivo do feijoeiro sem a aplicação desse nutriente. Nos tratamentos em que não houve aplicação de enxofre, não foram observadas reduções nos teores de $\mathrm{S}_{-} \mathrm{SO}_{4}^{-2}$ no solo com os cultivos sucessivos. Este fato pode ser decorrente da mineralização do enxofre orgânico do solo, graças às altas temperaturas e à umidade adequada nos vasos dentro da casa de vegetação, resultando na diminuição da matéria orgânica.

Na cultura do gergelim, a reaplicação das doses iniciais de enxofre permitiu o aumento linear no teor de $\mathrm{S}_{-} \mathrm{SO}_{4}^{-2}$ disponível na média dos quatro solos (teor de $\mathrm{S}-\mathrm{SO}_{4}^{-2}=10,04+0,408$ dose; $\mathrm{R}^{2}=0,99$ ). Assim, independentemente do tipo de solo, pois não houve interação significativa entre tipos de solos e doses de sulfato, para cada $\mathrm{kg}$ de $\mathrm{S}_{-} \mathrm{SO}_{4}^{-2}$ aplicado, $0,41 \mathrm{~kg}$ foi recuperado pelo método de $\mathrm{Ca}\left(\mathrm{H}_{2} \mathrm{PO}_{4}\right)_{2}$ na camada 0 $10 \mathrm{~cm}$. No entanto, os teores médios (média das doses) de $\mathrm{S}_{-} \mathrm{SO}_{4}^{-2}$ extraídos nos Latossolos (LVdf e LVAd) foram maiores $\left(20,6\right.$ e $\left.23,1 \mathrm{mg} \mathrm{dm}^{-3}\right)$ do que no PVd $(16,8 \mathrm{mg}$ $\mathrm{dm}^{-3}$ ), enquanto o teor médio encontrado no RQo foi de apenas $12,9 \mathrm{mg} \mathrm{dm}^{-3}$. Nesse solo, a disponibilidade de sulfato foi maior do que as observadas nos cultivos anteriores (Tabela 3), em conseqüência da reaplicação da dose de $\mathrm{S}$ e da possível mineralização de S orgânico proporcionada pelas altas temperaturas. Esse aumento da disponibilidade de $\mathrm{S}_{-} \mathrm{SO}_{4}^{-2}$ no solo mais arenoso pode também ser explicado pelo elevado consumo de água pelo gergelim, o que teria ocasionado a ascensão de água das camadas mais profundas do vaso, trazendo o S-SO ${ }_{4}^{-2}$ para a camada superficial. Embora em menor escala, nos outros três solos, também houve aumento nos teores médios de sulfato em relação à cultura anterior.

Após a cultura do trevo vesiculoso, onde foi reaplicado $1 / 3$ da dose inicial, também não houve interação significativa entre tipo de solo e doses de sulfato. Os teores médios (média das doses) de S-SO ${ }^{2}$ extraídos foram relacionados positivamente aos teores de argila dos solos, cujos valores foram de 24,7, 17,5, 14,2 e $10,9 \mathrm{mg} \mathrm{dm}^{-3} \mathrm{de} \mathrm{S} \mathrm{SO} \mathrm{S}^{-2}$ para os solos LVdf, LVAd, PVd e RQo, respectivamente (Tabela 3). Como os solos não receberam aplicação de $\mathrm{S}_{-} \mathrm{SO}_{4}^{-2}$ na semeadura do trigo, pode-se observar uma queda no teor médio de S$\mathrm{SO}_{4}^{-2,}$ se comparado ao cultivo anterior, principalmente em PVd e RQo, solos de baixo poder tampão de sulfato. Adicionalmente, houve interação significativa entre tipo de solo e dose de sulfato. No tratamento testemunha, os maiores teores de $\mathrm{S}_{-} \mathrm{SO}_{4}^{-2}$ foram extraídos dos Latossolos (12,9 e $10,0 \mathrm{mg} \mathrm{dm}^{-3}$, respectivamente, para LVdf e LVAd), seguido do PVd $\left(7,9 \mathrm{mg} \mathrm{dm}^{-3}\right)$ e do RQo $\left(4,0 \mathrm{mg} \mathrm{dm}^{-3}\right)$. Nos tratamentos que haviam recebido doses de sulfato, o LVdf manteve teores de $\mathrm{S}_{-} \mathrm{SO}_{4}^{-2}$ mais elevados do que os demais solos, inclusive para o outro Latossolo.

Com exceção do gergelim, todas as demais culturas testadas neste estudo apresentaram aumento no teor de enxofre nos tecidos da parte aérea e na quantidade exportada deste nutriente decorrentes da aplicação de sulfato (Tabela 4). Esse fenômeno, conhecido como "consumo de luxo", ocorre pela absorção de nutrientes em quantidades maiores que as demandadas pelo metabolismo da planta. Os íons absorvidos em excesso geralmente são compartimentalizados nos vacúolos das células. Em todas as culturas testadas, em quaisquer que fossem os tratamentos, o teor de $\mathrm{S}$ no tecido foi igual ou superior ao teor considerado como crítico $(0,10-0,25 \%)$ por KAMPRATH \& TILL (1983), AMBROSANO et al. (1996) e EMBRAPA (1998). No cultivo da canola, não houve interação significativa entre tipo de solos e doses de sulfato. Os maiores teores de $\mathrm{S}$ no tecido foram obtidos no solo RQo $(0,63 \%)$, e os menores nos LVAd $(0,38 \%)$ e no PVd $(0,35 \%)$. Isso pode ser explicado pela menor produção de matéria seca proporcionada no RQo, promovendo um maior efeito de concentração de $\mathrm{S}$ no tecido. Além disso, esses maiores teores de $\mathrm{S}$ no tecido da canola cultivada no solo RQo podem ser devidos á maior concentração de $\mathrm{S}$ na solução do solo e pela menor capacidade de adsorção deste solo. Como os vasos são fechados no fundo, não havendo saída de água e lixiviação de nutrientes, este enxofre disponível não foi perdido do sistema, sendo absorvido pela planta. A máxima produção de matéria seca de canola em função da aplicação de enxofre nos solos LVdf, LVAd e PVd correspondeu a teores próximos a $0,43 \%$ de $\mathrm{S}$ no tecido. Estes teores foram bem superiores àquele teor, acima do qual, segundo MALAVOLTA (1984) e MENGEL \& KIRKBY (1987), a planta apresentaria pouca possibilidade de resposta à aplicação desse nutriente. Embora houvesse interação significativa entre tipo de solo e dose de sulfato quanto aos teores de $\mathrm{S}$ no tecido das culturas de soja, feijoeiro, gergelim e trevo vesiculoso, a quantidade exportada pela parte aérea foi independente da interação. Os valores médios (média das doses) das quantidades exportadas encontram-se na tabela 4 , onde, por exemplo, as maiores quantidades exportadas pela cultura da soja foram observadas no RQo $\left(29,1 \mathrm{mg} \mathrm{vaso}^{-1}\right)$ e no PVd (28,1 mg vaso-1), comparativamente com os Latossolos (17,9 e 17,6 mg vaso ${ }^{-1}$, para o LVdf e LVAd, respectivamente). 
Tabela 4 - Teor de S no tecido da parte aérea e exportação deste nutriente por seis cultivos em sucessão em decorrência de doses de enxofre em quatro diferentes tipos de solos.

\begin{tabular}{|c|c|c|c|c|c|c|c|c|}
\hline \multirow{2}{*}{$\begin{array}{l}\text { Doses } \\
\text { acumuladas } \\
\text { de } \mathrm{S}_{-} \mathrm{SO}_{4}{ }^{-2}\end{array}$} & \multicolumn{2}{|c|}{$\mathrm{LVdf}^{1}$} & \multicolumn{2}{|c|}{ LVAd } & \multicolumn{2}{|c|}{$\mathrm{PVd}$} & \multicolumn{2}{|c|}{ RQo } \\
\hline & S no tecido & $\begin{array}{c}\text { Exportação } \\
\text { de S }\end{array}$ & S no tecido & $\begin{array}{c}\text { Exportação } \\
\text { de S }\end{array}$ & S no tecido & $\begin{array}{c}\text { Exportação } \\
\text { de S }\end{array}$ & S no tecido & $\begin{array}{c}\text { Exportação } \\
\text { de S }\end{array}$ \\
\hline $\mathrm{mg} \mathrm{vaso}^{-1}$ & $\%$ & $\mathrm{mg} \mathrm{vaso}^{-1}$ & $\%$ & $\mathrm{mg} \mathrm{vaso}^{-1}$ & $\%$ & $\mathrm{mg} \mathrm{vaso}^{-1}$ & $\%$ & $\mathrm{mg} \mathrm{vaso}^{-1}$ \\
\hline \multicolumn{9}{|c|}{...Canola. } \\
\hline 0 & 0,11 & $14,2 \mathrm{a}^{3}$ & 0,12 & $10,9 \mathrm{a}$ & 0,09 & $6,4 \mathrm{a}$ & 0,25 & $17,9 \mathrm{a}$ \\
\hline 55 & 0,31 & $41,7 \mathrm{a}$ & 0,31 & $37,4 \mathrm{ab}$ & 0,21 & $22,8 \mathrm{~b}$ & 0,66 & $28,3 \mathrm{ab}$ \\
\hline 110 & 0,47 & $65,5 \mathrm{a}$ & 0,43 & $64,0 \mathrm{a}$ & 0,41 & $37,2 \mathrm{~b}$ & 0,73 & $26,7 \mathrm{~b}$ \\
\hline 220 & 0,71 & $105,6 \mathrm{a}$ & 0,66 & $102,2 \mathrm{a}$ & 0,69 & $72,8 \mathrm{~b}$ & 0,86 & $40,8 \mathrm{c}$ \\
\hline Média & $0,40 \mathrm{~B}^{2}$ & & $0,38 \mathrm{BC}$ & & $0,35 \mathrm{C}$ & & $0,63 \mathrm{~A}$ & \\
\hline $\mathrm{CV} \%$ & \multicolumn{6}{|c|}{$\mathrm{S}$ no tecido $=15,7$ e exportação de $\mathrm{S}=25,9$} & & \\
\hline & & & & & oja........... & & & \\
\hline 0 & $0,10 \mathrm{C}$ & 10,0 & $0,10 \mathrm{C}$ & 10,3 & $0,26 \mathrm{~A}$ & 21,4 & $0,19 \mathrm{~B}$ & 18,3 \\
\hline 73,3 & $0,17 \mathrm{~B}$ & 16,9 & $0,16 \mathrm{~B}$ & 15,2 & $0,31 \mathrm{~A}$ & 27,0 & $0,32 \mathrm{~A}$ & 28,8 \\
\hline 146,6 & $0,25 \mathrm{~B}$ & 21,9 & $0,22 \mathrm{~B}$ & 22,5 & $0,35 \mathrm{~A}$ & 31,5 & $0,34 \mathrm{~A}$ & 28,8 \\
\hline 293,3 & $0,24 \mathrm{C}$ & 22,7 & $0,23 \mathrm{C}$ & 22,3 & $0,35 \mathrm{~B}$ & 32,5 & $0,47 \mathrm{~A}$ & 40,4 \\
\hline Média & & $17,9 b$ & & $17,6 b$ & & $28,1 \mathrm{a}$ & & $29,1 \mathrm{a}$ \\
\hline $\mathrm{CV} \%$ & \multicolumn{6}{|c|}{$\mathrm{S}$ no tecido $=12,9$ e exportação de $\mathrm{S}=17,7$} & & \\
\hline 0 & $\begin{array}{c}\ldots \ldots \ldots \ldots \\
0,27 \mathrm{~A}\end{array}$ & $\begin{array}{c}\ldots \ldots \ldots \\
30,1\end{array}$ & $\begin{array}{c}\ldots \ldots \ldots \\
0,25 \mathrm{~A}\end{array}$ & $\begin{array}{l}\ldots \ldots \ldots \\
29,9\end{array}$ & $\begin{array}{c}\text { ro.......... } \\
0,17 \mathrm{~B}\end{array}$ & 16,7 & $0,28 \mathrm{~A}$ & 21,6 \\
\hline 73,3 & $0,28 \mathrm{~B}$ & 33,4 & $0,26 \mathrm{~B}$ & 30,2 & $0,25 \mathrm{~B}$ & 23,7 & $0,33 \mathrm{~A}$ & 25,1 \\
\hline 146,6 & $0,29 \mathrm{~B}$ & 33,5 & $0,34 \mathrm{~A}$ & 38,9 & $0,28 \mathrm{~B}$ & 28,1 & $0,34 \mathrm{~A}$ & 28,9 \\
\hline 293,3 & $0,36 \mathrm{~A}$ & 40,3 & $0,37 \mathrm{~A}$ & 42,4 & $0,35 \mathrm{~A}$ & 36,9 & $0,35 \mathrm{~A}$ & 31,1 \\
\hline Média & & $34,3 \mathrm{a}$ & & $35,3 \mathrm{a}$ & & $26,3 b$ & & $26,7 b$ \\
\hline \multirow[t]{2}{*}{$\mathrm{CV} \%$} & \multicolumn{6}{|c|}{$\mathrm{S}$ no tecido $=11,5$ e exportação de $\mathrm{S}=17,7$} & & \\
\hline & & & & & gelim......... & ........ & ........... & \\
\hline 0 & $0,31 \mathrm{~A}$ & 24,7 & $0,29 \mathrm{~A}$ & 20,2 & $0,26 \mathrm{~A}$ & 18,1 & $0,28 \mathrm{~A}$ & 17,2 \\
\hline 128,3 & $0,31 \mathrm{~B}$ & 25,6 & $0,33 \mathrm{AB}$ & 23,3 & $0,36 \mathrm{~A}$ & 21,8 & $0,30 \mathrm{~B}$ & 16,9 \\
\hline 256,7 & $0,31 \mathrm{~B}$ & 26,5 & $0,30 \mathrm{~B}$ & 17,6 & $0,40 \mathrm{~A}$ & 26,5 & $0,29 \mathrm{~B}$ & 14,1 \\
\hline 513,3 & $0,28 \mathrm{~B}$ & 23,0 & $0,30 \mathrm{~B}$ & 23,1 & $0,40 \mathrm{~A}$ & 23,8 & $0,31 \mathrm{~B}$ & 17,2 \\
\hline Média & & $24,9 \mathrm{a}$ & & $21,0 \mathrm{~b}$ & & $22,5 \mathrm{ab}$ & & $16,3 \mathrm{c}$ \\
\hline $\mathrm{CV} \%$ & \multicolumn{6}{|c|}{$\mathrm{S}$ no tecido $=10,8$ e exportação de $\mathrm{S}=19,3$} & & \\
\hline 0 & $0,30 \mathrm{~A}$ & 25,6 & $0,28 \mathrm{~A}$ & 36,6 & $\begin{array}{c}\ldots \ldots \ldots \ldots \\
0,25 \mathrm{~A}\end{array}$ & 34,7 & $0,28 \mathrm{~A}$ & 37,5 \\
\hline 146,6 & $0,36 \mathrm{~A}$ & 38,3 & $0,38 \mathrm{~A}$ & 49,6 & $0,39 \mathrm{~A}$ & 49,2 & $0,35 \mathrm{~A}$ & 46,8 \\
\hline 293,4 & $0,37 \mathrm{BC}$ & 38,7 & $0,45 \mathrm{~A}$ & 63,2 & $0,43 \mathrm{AB}$ & 54,8 & $0,32 \mathrm{C}$ & 41,5 \\
\hline 586,6 & $0,46 \mathrm{~B}$ & 48,7 & $0,50 \mathrm{AB}$ & 64,7 & $0,49 \mathrm{AB}$ & 71,4 & $0,54 \mathrm{~A}$ & 64,9 \\
\hline Média & & $37,8 \mathrm{~b}$ & & $53,5 \mathrm{a}$ & & $52,5 \mathrm{a}$ & & $47,7 \mathrm{a}$ \\
\hline $\mathrm{CV} \%$ & \multicolumn{6}{|c|}{$\mathrm{S}$ no tecido $=13,2$ e exportação de $\mathrm{S}=18,0$} & & \\
\hline 0 & 0,12 & 12,4 & 0,16 & 16,7 & 0,20 & 20,2 & 0,27 & 20,5 \\
\hline 146,6 & 0,23 & 24,2 & 0,21 & 23,4 & 0,31 & 37,7 & 0,36 & 30,2 \\
\hline 293,4 & 0,26 & 27,5 & 0,29 & 33,9 & 0,35 & 35,7 & 0,36 & 39,5 \\
\hline 586,6 & 0,32 & 36,2 & 0,34 & 37,7 & 0,45 & 49,1 & 0,41 & 43,5 \\
\hline Média & $0,23 \mathrm{~B}$ & $25,1 b$ & $0,25 \mathrm{~B}$ & $27,9 b$ & $0,33 \mathrm{~A}$ & $35,7 \mathrm{a}$ & $0,35 \mathrm{~A}$ & $33,4 \mathrm{a}$ \\
\hline $\mathrm{CV} \%$ & \multicolumn{6}{|c|}{$\mathrm{S}$ no tecido $=11,3$ e exportação de $\mathrm{S}=15,5$} & & \\
\hline
\end{tabular}

${ }^{1}$ Latossolo Vermelho-Amarelo Distrófico típico (LVAd); e Latossolo Vermelho Distroférrico típico (LVdf); Argissolo Vermelho Distrófico arênico (PVd) e Neossolo Quatzarênico Órtico típico (RQo).

${ }^{2}$ Médias dos teores de $\mathrm{S}$ no tecido seguidas pela mesma letra maiúscula na linha, comparando as classes de solo dentro de cada dose de S$\mathrm{SO}_{4}^{-2}$ ou na média das doses, não diferiram estatisticamente pelo teste de Tukey $(\alpha 5 \%)$.

${ }^{3}$ Médias das quantidades exportadas de $\mathrm{S}$ seguidas pela mesma letra minúscula na linha, comparando as classes de solo dentro de cada dose de $\mathrm{S}_{-} \mathrm{SO}_{4}{ }^{-2}$ ou na média das doses, não diferiram estatisticamente pelo teste de Tukey ( $\left.\alpha 5 \%\right)$. 
Analisando os resultados obtidos neste experimento, pode-se considerar que a dinâmica do $\mathrm{S}$ em diferentes tipos de solo e os fatores que regulam sua disponibilidade às plantas são bastante complexos, dificultando a utilização do atual método de análise de $\mathrm{S}$ disponível, especialmente da camada superficial do solo no sistema plantio direto, para fins de diagnóstico e recomendação da adubação sulfatada.

\section{CONCLUSÕES}

A cultura da canola respondeu em produção de matéria seca à aplicação de enxofre, não ocorrendo respostas para as culturas da soja, do feijoeiro, do gergelim, do trevo vesiculoso e do trigo. Exceto para a canola, não houve relação entre o rendimento relativo de matéria seca e os teores de $\mathrm{S}_{-} \mathrm{SO}_{4}^{-2}$ da camada de 0 $10 \mathrm{~cm}$.

Com exceção da cultura do gergelim, o teor de enxofre no tecido da parte área e a exportação deste nutriente pelas culturas aumentaram com a fertilização sulfatada, mesmo nas culturas que não apresentaram aumentos em produção de matéria seca. Elevadas adições de enxofre no solo podem significar maior absorção pela planta, sem que ocorram correspondentes aumentos na produção de matéria seca.

\section{REFERÊNCIAS}

AMBROSANO, E.J. et al. Leguminosas e oleaginosas. In: RAIJ, B. van et al. (Eds). Recomendações de adubação e calagem para o estado de São Paulo, 2. Campinas: Instituto Agronômico, Fundação IAC, 1996. p.187-203.

ANGHINONI, I. Resposta da cultura da soja a aplicação de boro, zinco e enxofre (solo Camaquã). In: REUNIÃO CONJUNTA DE PESQUISA DA SOJA-RS/SC, 3., 1975, Porto Alegre. Anais... Porto Alegre: FECOTRIGO, 1975. p.126.

ANGHINONI, I. et al. Respostas da cultura da soja à aplicação de boro, zinco e enxofre. Agronomia Sulriograndense, v.12, p.189-99, 1976.

BISSANI, C.A. Disponibilidade de S para as plantas em solos do Rio Grande do Sul. 1985. 198f. Dissertação (Mestrado em Ciência do Solo) - Programa de Pós-graduação em Agronomia, Universidade Federal do Rio Grande do Sul.

CASAGRANDE, J.C. et al. Adsorção de fosfato e sulfato em solos com cargas elétricas variáveis. Revista Brasileira de Ciência do Solo, v.27, p.51-59, 2003.

CHAO, T.T. et al. Soil constituents and properties in the absorption of sulfate ions. Soil Science, v.94, p.276-286, 1962.
CQFS-RS/SC. COMISSÃO DE QUÍMICA E FERTILIDADE DO SOLO - RS/SC. Manual de adubação e de calagem para os estados do Rio Grande do Sul e Santa Catarina. Porto Alegre: SBCS - Núcleo Regional Sul, 2004. 400p.

COSTA, C.A.S. Mineralização do S orgânico e adsorção de sulfato em solos. 1980. 65f. Dissertação (Mestrado em Agronomia) - Programa de Pós-graduação em Agronomia, Universidade Federal do Rio Grande do Sul.

ELKINS, D.M.; ELMINGER, L.E. Effect of soil pH on the availability of adsorbed sulfate. Soil Science Society American Proceeding, v.35, p.931-934, 1971.

EMBRAPA Centro Nacional de Pesquisas de Soja. Recomendações técnicas para a cultura da soja na região central do Brasil. Londrina: Embrapa-CNPSoja, 1998. 182p.

ERIKSEN, J.; ASKEGAARD, M. Sulphate leaching in an organic crop rotation on sandy soil in Denmark. Agriculture, Ecosystems \& Environment, v.78, n.1, p.107-114, 2000.

FRENEY, J.R. et al. Soil organic matter fractions as sources of plant-available sulfur. Soil Biology and Biochemistry, v.7, p.217-221, 1975.

GOEPFERT, C.F.; KUSSOW, W.F. A necessidade de aplicar S e microelementos em oito solos do Rio Grande do Sul. Agronomia Sulriograndense, v.7, n.2, p.149-156, 1971.

KAMPRATH, E.J.; TILL, A.R. Sulfur cycling in the tropic. In: BLAIR, G.J; TILL, A.R. (Ed). Sulfur in South-east Asian and South Pacific agriculture. Washington: The Australian Development Assistance Bureau \& The Sulfur Institute, 1983. p.1-14.

KORENTAJER, L. et al. The effect of liming and leaching on the sulfur supplying capacity of soil. Soil Science Society American Journal, v.47, p.525-530, 1983.

LARBIER, M.; LECLERCQ, B. Nutrition and Feeding of poultry. Nottingham: Nottingham University, 1992. 305p.

MALAVOLTA, E. Efeitos de doses e fontes de enxofre em culturas de interesse econômico. I- Capim colonião. IIColza. III- Sorgo Sacarino. São Paulo: Centro de Pesquisa e promoção de Sulfato de Amônio, 1984. 60p. (Boletim técnico, 3).

MENGEL, K.; KIRKBY, E.A. Principles of plant nutrition. 3.ed. Bern: Potash Institute, 1987. 687p.

MIELNICZUK, J. et al. Interações fertilidade e conservação do solo. In: FERTBIO 2000. Santa Maria: SBCS, 2000. (CDRom). $15 \mathrm{p}$.

NACIMENTO, J.A.L.; MORELLI, M. Enxofre em solos do Rio Grande do Sul. I. Formas no solo. Revista Brasileira de Ciência do Solo, v.4, p.131-135, 1980.

NEPTUNE, A.M.L. et al. Sulfur fractions and carbon-nitrogenphosphorus-sulfur relationship in same Brazilian and Iowa soils. 
Soil Science Society American Proceeding, v.39, n.1, p.51$55,1975$.

PARFITT, R.L.; SMART, R.S.C. The mechanism of sulfate absorption of iron oxides. Soil Science Society American Journal, v.42, p.48-50, 1978.

RHEINHEIMER, D.S. et al. Situação da fertilidade dos solos no Estado do Rio Grande do Sul. Santa Maria: Universidade Federal de Santa Maria, Departamento de Solos, 2001. 41p. (Boletim técnico, 2).

SOLOMONS, D. et al. Sulphur speciation and biogeochemical cycling in long-term arable cropping of subtropical soils: evidence from wet-chemical reduction and S K-edge XANES spectroscopy. European Journal of Soil Science, v.56, p.621-634, 2005.

TEDESCO, M.J. et al. Análise de solo, plantas e outros materiais. 2.ed. Porto Alegre: UFRGS, 1995. 215p. (Boletim Técnico, 5).

WOLFFENBÜTTEL, R.; TEDESCO, M.J. Disponibilidade de S para alfafa em oito solos do Rio Grande do Sul e sua relação com parâmetro do solo. Agronomia Sulriograndense, v.17, p.357-376, 1981 